\title{
Channel Phase Error Compensation for MIMO-SAR
}

\author{
Lei Zhang, ${ }^{1,2}$ Yunkai Deng, ${ }^{1}$ and Robert Wang ${ }^{1}$ \\ ${ }^{1}$ Department of Spaceborne Microwave Remote Sensing System, Institute of Electronics, Chinese Academy of Sciences, \\ Beijing 100190, China \\ ${ }^{2}$ University of Chinese Academy of Sciences, Chinese Academy of Sciences, Beijing 100190, China
}

Correspondence should be addressed to Lei Zhang; 314forever@163.com

Received 27 February 2014; Accepted 2 June 2014; Published 22 June 2014

Academic Editor: Wen-Qin Wang

Copyright (C) 2014 Lei Zhang et al. This is an open access article distributed under the Creative Commons Attribution License, which permits unrestricted use, distribution, and reproduction in any medium, provided the original work is properly cited.

Multi-input multioutput (MIMO) is a novel technique to achieve high-resolution as well as wide swath in synthetic aperture radar (SAR) systems. Channel imbalance is inevitable in multichannel systems that it declines the imaging quality. Generally, the imbalance cannot be fully compensated by simple internal calibration in a MIMO-SAR system. In this paper, a new algorithm based on raw data is presented to remove the channel phase error. Based on the error source, this approach models the phase error as two parts: the transmit phase error and the receive phase error. The receive phase error is removed using cost function at the azimuth processing stage, whereas the transmit phase error is estimated with correlation. Point target simulations confirm the influence of channel phase error and the validation of the proposed approach. Besides, the performance is also investigated.

\section{Introduction}

Synthetic aperture radar (SAR) is a powerful microwave instrument for remote sensing [1]. 2D even 3D [2] image of the terrestrial surface can be obtained independent of weather and sunlight illumination. High-resolution as well as wide swath (HRWS) is one of the main goals of the system engineers $[3,4]$.

For the sake of high azimuth resolution, people have developed spotlight mode $[5,6]$ in which the antenna points toward the same region during the whole data acquisition. While obtaining large imaging swath, ScanSAR mode [7] is proposed in which the antenna directivity is fixed, swapping between different strips. Limited by the minimum-antennaarea constraint [1], conventional spaceborne SAR is hard to achieve high-resolution in azimuth meanwhile wide imaging swath. High azimuth resolution requires large Doppler bandwidth, which means a high pulse repetition frequency (PRF) to comply with the Nyquist theory, whereas, wide imaging swath demands wide range beam width in which the PRF must be low to guarantee the echo's completeness. Modes such as sliding-spotlight and TOPS $[8,9]$ are the trade-off between the two demands.
In range direction, SAR transmits chirp pulse. The resolution is proportional to the signal bandwidth, which is defined as

$$
\sigma_{r}=\frac{c}{2 B}
$$

where $\sigma_{r}$ is the range resolution, $c$ is the velocity of light, and $B$ is the signal bandwidth. For $0.1 \mathrm{~m}$ resolution in slant range, $1.5 \mathrm{GHz}$ bandwidth is necessary. This implies at least $1.8 \mathrm{GHz}$ sampling rate (with oversampling rate $20 \%$ ). The high quality, that is, radiation hardened, $\mathrm{A} / \mathrm{D}$ convertor is quite difficult to manufacture.

Multi-input multioutput (MIMO) technique provides an opportunity to break through these constraints [10,11]. The antenna being divided into several apertures, each aperture transmits pulses at different center frequencies simultaneously and all apertures receive the echoes. In this way, numerous effective phase centers are formed. These effective phase centers may be overlapped in spacetime or in frequency. Then using the signal processing technique signals with large bandwidth (both in range and in Doppler) as well as wide swath are obtained $[12,13]$. 
Channel imbalance is inevitable in multichannel system due to the effects of the qualities of modules, the construction of the system, and the atmosphere. The imbalance presents in echo data in terms of amplitude error and phase error. These errors impair the signal synthesis in which the final SAR image will be ambiguous even unrecognized.

Channel imbalance can be removed by means of system internal calibration [1] or analyzing through raw data. Methods based on internal calibration are precise but cannot calibrate the phase errors caused by the antenna, whereas literature belonging to the latter is rare to see. In this paper, an algorithm based on raw data to handle channel phase imbalance in MIMO-SAR system is proposed. The method models phase imbalance as two parts: the transmit phase error (TXE) and the receive phase error (RXE). The transmit phase error is estimated by correlation operation, whereas the receive phase error is removed using cost function at the azimuth processing stage.

This paper is organized as follows. In Section 2, the signal mode is introduced. The proposed method is presented in Section 3 with simulations and discussions in Section 4. Section 5 will give the conclusion.

\section{Signal Mode}

Figure 1 is a diagram of a MIMO-SAR system with five channels in azimuth. Antennas of each channel and the corresponding phase-centers are marked with rectangles and triangles, respectively. Each channel transmits pulse simultaneously and receives the echoes transmitted by not only itself but also the others. Suppose channel $m$ transmits pulse at center frequency $f_{c, m}$. By compensating a known constant phase between channels, the received echo-signal can be converted into the equivalent self-transmit and selfreceive signal [14]. In Figure 1(b), the effective phase-centers are marked with circles which are located halfway in between the transmitting and the respective receiving phase-centers. The effective phase-centers of different center frequencies are marked with distinct colors. Theoretically, if a MIMO-SAR transmits pulse with $M$ channels and receives with $N$, the total effective phase-centers is $M \cdot N$.

The signal transmitted by channel $m$ is

$$
s_{t, m}(\tau, \eta)=\operatorname{rect}\left(\frac{\tau}{T}\right) \exp \left\{j 2 \pi\left(f_{c, m} \tau+\frac{1}{2} K_{r} \tau^{2}\right)\right\} \text {, }
$$

where $\tau$ is the fast time, $\eta$ is the slow time, $T$ is the pulse length, and $K_{r}$ is the chirp rate. Then the signal after mixing with center frequency $f_{c, m}$ received by channel $n$ is

$$
\begin{aligned}
s_{r, n, m}(\tau, \eta)= & \sigma \cdot \operatorname{rect}\left(\frac{\tau-2 R_{n, m}(\eta) / c}{T}\right) \\
& \times \exp \left\{-j \frac{4 \pi R_{n, m}(\eta) f_{c, m}}{c}\right\} \\
& \times \exp \left\{j \pi K_{r}\left(\tau-\frac{2 R_{n, m}(\eta)}{c}\right)^{2}\right\},
\end{aligned}
$$

where $\sigma$ is the backscattering coefficient of the target, $c$ is the velocity of light, and $R_{n, m}(\eta)$ is the slant range

$$
R_{n, m}(\eta)=\sqrt{R_{0}^{2}+\left(V_{r} \eta+\frac{(n-1) \cdot d}{2}+\frac{(m-1) \cdot d}{2}\right)^{2}}
$$

where $R_{0}$ is the nearest range, $V_{r}$ is the platform velocity, $d$ is the antenna length in azimuth of a single channel, and $m$ and $n$ ranges from 1 to $M$. In (4), the reference channel is channel 1 ; that is, the reference slant range is $R_{1,1}(\eta)=\sqrt{R_{0}^{2}+V_{r}^{2} \eta^{2}}$.

Consider the signals with a specific center frequency. They can be treated as the azimuth multichannel SAR signals. Usually, all channels have the same the antenna lengths. According to the slow time delay, after compensating the known constant phase, the received signals have

$$
s_{r, n, m}(\tau, \eta)=s_{r, 1, m}\left(\tau, \eta+\frac{(n-1) \cdot d}{2 V_{r}}\right) .
$$

If the radar platform velocity and the PRF have a relationship

$$
\mathrm{PRF}=\frac{M d}{2 V_{r}}
$$

where $M$ is the number of receive channels and $d$ is the antenna length of a single channel, the effective phase-centers are distributed uniformly in azimuth. However, (6) is hard to meet strictly in practical SAR systems because of the timing restriction, and therefore the effective phase-centers are nonuniform distribution as the upper part of Figure 1(b) shows. Using the signal processing technique such as system filters [14] or STAP [15] (the matrices are identical indeed), the data can be reconstructed as uniformly sampled. The lower part of Figure 2(b) is the effective phase-centers after reconstruction where the dashed line denotes the zero delay. It is interesting that the time delays are different for each $f_{c, m}$ because (4) associates with $m$.

From Figure 1(b), it can be seen that the effective phasecenters of different $f_{c, m}$ are overlapped after reconstruction. If the pulse bandwidth of all transmit channels is identical and $f_{c, m}$ steps following

$$
f_{c, m}-f_{c, m-1} \leq B
$$

where $B$ is the chirp bandwidth, then a chirp signal with large bandwidth can be obtained using the subband synthesis technique with the overlapped samples [16]. Subband synthesis can be realized either in the range time domain or in the range frequency domain. Besides, synthesis can be before range compression or after compression.

In summary, the MIMO-SAR processing steps followed by this paper are

(1) samples reconstruction in azimuth;

(2) imaging each subband;

(3) subband synthesis in range. 


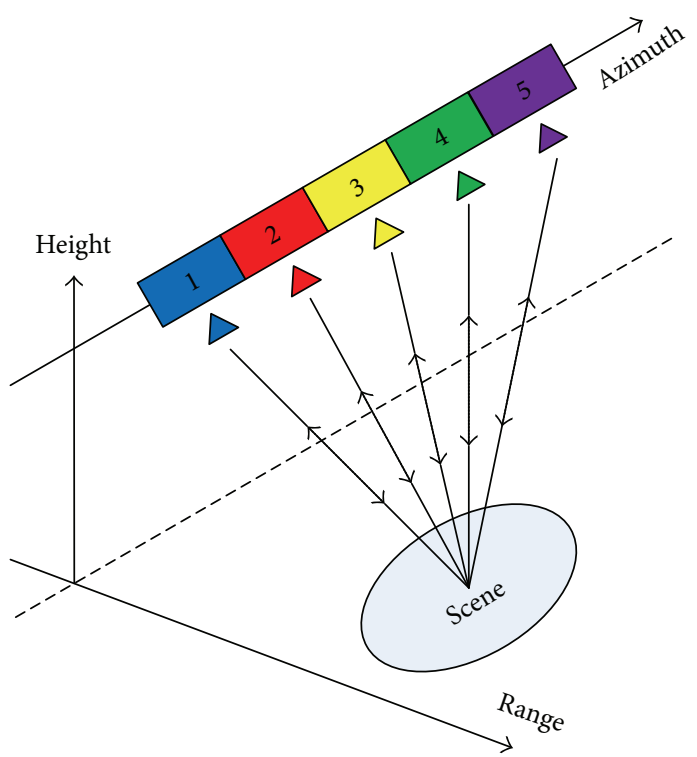

(a)

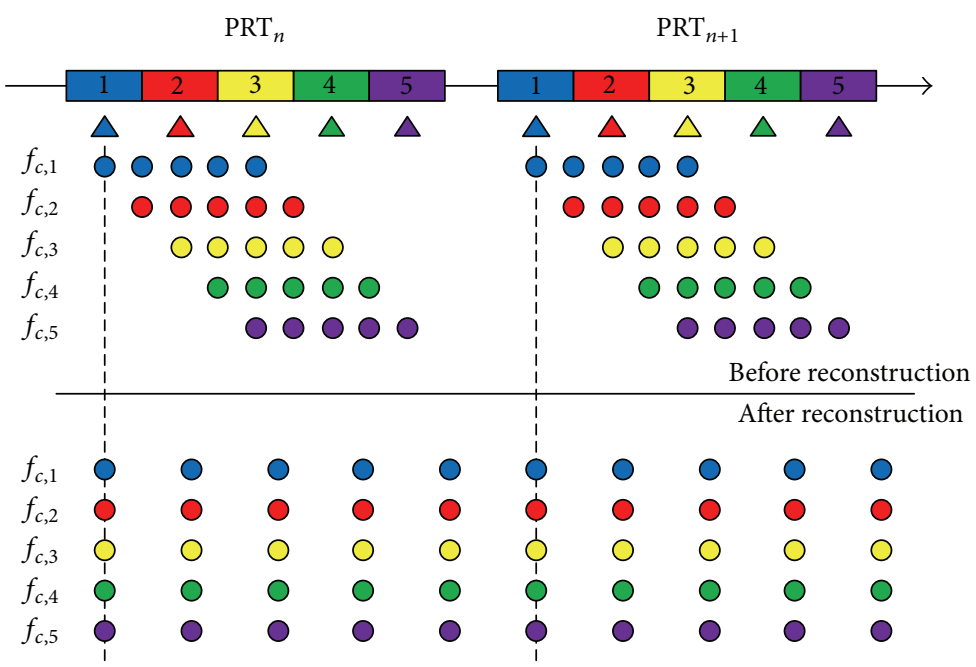

(b)

FIGURE 1: Diagram of a MIMO-SAR system. (a) System geometry. (b) Phase sequence.

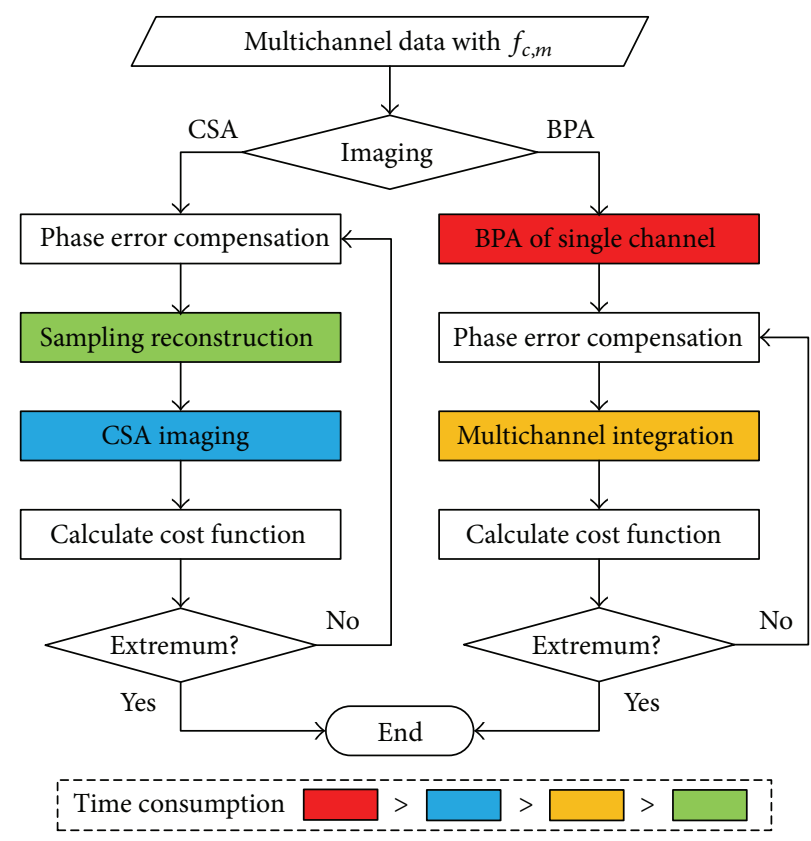

FIGURE 2: Flow chart of removing RXE.

\section{Phase Error and the Compensation}

Channel imbalance is inevitable in multichannel system, not expecting MIMO-SAR. It presents in echo data in terms of amplitude error and phase error. We only focus on phase error in this paper. Denote the transmission characteristic of each transmit channel as $\Delta \psi_{1}, \Delta \psi_{2}, \ldots, \Delta \psi_{M}$ and each receive channel as $\Delta \varphi_{1,1}, \Delta \varphi_{1,2}, \ldots, \Delta \varphi_{1, M}, \Delta \varphi_{2,1}, \Delta \varphi_{2,2}$
$, \ldots, \Delta \varphi_{2, M}, \ldots, \Delta \varphi_{M, 1}, \Delta \varphi_{M, 2}, \ldots, \Delta \varphi_{M, M}$, respectively. Here, the substrip of $\Delta \psi$ indicates the transmit channel number, and $\Delta \varphi_{n, m}$ indicates the characteristic of channel $n$ receiving the signals with center frequency $f_{c, m}$. The actual signals received are

$$
s_{r, n, m}(\tau, \eta)=\Delta \psi_{m} \cdot \Delta \varphi_{n, m} \cdot s_{r, 1, m}\left(\tau, \eta+\frac{(n-1) \cdot d}{2 V_{r}}\right) .
$$


3.1. The Receive Phase Errors. Consider the signals with center frequency $f_{c, m}$ and suppose channel 1 is the reference channel. Define the phase error

$$
\Delta \varepsilon_{r, n, m}=\frac{\Delta \varphi_{n, m}}{\Delta \varphi_{1, m}}, \quad n=2, \ldots, M
$$

the receive phase error ( $\mathrm{RXE}$ ). RXE is range invariant and if arranged along azimuth, it periodically changes over a cycle of $M$ samples. It can be treated as some form of phase error introduced by motion errors in airborne SAR system; therefore RXE brings blurring on subband imaging that the images are ambiguous and defocused in the azimuth.

The compensation of RXE can be treated as a special case of autofocus. There are numerous autofocus methods; however not all are suitable for RXE removing. Because the azimuth reconstruction will disturb the phase error, the phase estimated by autofocus is usually not the original one. We resort to the method using cost function [17] in which the phase error is trial rather than directly derived from the imagery.

Generally, the value of cost function relates to the extent of an image into focus. The broaden mainlobe of a point target and the ghosts along azimuth are the two main characteristics blurred images caused by RXE exhibited [18]. Although defocused, the ghosts share the same shape with the actual targets. According to these two characteristics, we develop a new cost function called image self-correlation in azimuth (ISCA):

$$
\mathrm{CF}_{\mathrm{ISCA}}(k)=\frac{E_{m}\left\{I(p, q) \cdot I^{*}(p, q+r)\right\}}{E_{m}\left\{I(p, q) \cdot I^{*}(p, q)\right\}},
$$

where $I(p, q)$ is a grey-scale SAR image, $p$ and $q$ are the range coordinate and the azimuth one, respectively, $r$ is the azimuth offset, * denotes conjugation, and $E_{m}$ implies average in range. ISCA is normalized by the denominator in which the maximum value is 1 which happens only when $k=0$.

ISCA describes the RXE in two ways. First, the ISCA of a focused image descends faster around $k=0$ (zero peak) than that of a defocused one; that is, the zero peak is sharper, because the mainlobe is wider on the defocused image. Second, besides the zero peak, the curve of selfcorrelation may have other couples of peaks (nonzero peak). When the image is fully focused, the ghosts are suppressed that these nonzero peaks become flat.

Thus, the estimation of RXE can be described as an optimization procedure

$$
\Delta \widehat{\boldsymbol{\varepsilon}}_{r, m}=\arg \underset{\Delta \boldsymbol{\varepsilon}_{r, m}}{\operatorname{ext}} \mathrm{CF}_{\mathrm{ISCA}},
$$

where $\Delta \widehat{\boldsymbol{\varepsilon}}_{r, m}$ is the optimal solution, $\Delta \boldsymbol{\varepsilon}_{r, m}=$ $\left[\Delta \varepsilon_{r, 2, m}, \Delta \varepsilon_{r, 3, m}, \ldots, \Delta \varepsilon_{r, M, m}\right]$, and operator ext denotes judging via the width of the zero peak or with the height of nonzero peaks of ISCA.

As the optimization in (11) has no closed-form solution, an iterative method called coordinate descent algorithm [19] is adopted in which the elements of $\Delta \boldsymbol{\varepsilon}_{r, m}$ are obtained in sequence. The iterative processes as well as the computational burden are distinctively different according to the imaging algorithm applied.

The iteration flow, when frequency domain imaging algorithm such as the familiar chirp scaling (CSA) [20] is chosen, is shown on the left of Figure 2. Because some time-consuming progresses are involved in iteration, the time consumption of the whole iteration will be quite large especially when the number of channels is big. This is the inherent limitation of cost function autofocusing.

Situations will turn better when time domain imaging algorithm such as back projection imaging algorithm (BPA) [21] is used. Integrating along the target track precisely, BPA can be used in almost all SAR working modes. Resembling the idea of fast factorized BP (FFBP), the data of each single channel are imaged first and then integrated as the right of Figure 2 shows. As only multichannel integration is involved in iteration, less time is required. After each single channel is imaged for the first time, we can select some range bins with highest contrast as a new data set for the iteration ahead. This will speed up the iteration further.

3.2. The Transmit Phase Errors. When RXE is compensated, we obtain $M$ frame focused images with different center frequencies and (8) becomes

$$
s_{r, n, m}^{\prime}(\tau, \eta)=\Delta \psi_{m} \cdot \Delta \varphi_{1, m} \cdot s_{r, 1, m}\left(\tau, \eta+\frac{(n-1) \cdot d}{2 V_{r}}\right) .
$$

Similarly, suppose channel 1 is the reference channel. Define the phase error

$$
\Delta \varepsilon_{t, m}=\frac{\Delta \psi_{m} \Delta \varphi_{1, m}}{\Delta \psi_{1} \Delta \varphi_{1, m}}, \quad m=2, \ldots, M
$$

the transmit phase error (TXE). TXE will reduce the performance of subband synthesis in which the peak sidelobe level ratio (PSLR) and the integrated sidelobe level ratio (ISLR) are worsened [22].

On the focused images, the target is compressed as

$$
\begin{aligned}
s_{c, n, m}(\tau, \eta)= & A \cdot \Delta \psi_{m} \cdot \Delta \varphi_{1, m} \cdot p_{r}\left(\tau-\frac{2 R_{n, m}(\eta)}{c}\right) p_{a}(\eta) \\
& \times \exp \left\{-j \frac{4 \pi R_{n, m}(\eta) f_{c, m}}{c}\right\} \exp \left\{j 2 \pi f_{\mathrm{dc}} \eta\right\},
\end{aligned}
$$

where $A$ is a irrespective coefficient, $p_{r}$ and $p_{a}$ are the amplitude of impulse response; that is, the sinc function, $f_{\mathrm{dc}}$ is the Doppler center. $R_{n, m}(\eta)$ is known as (4); so the estimation of TXE is simple compared to RXE in which

$$
\begin{aligned}
\Delta \varepsilon_{t, m}= & \frac{s_{c, n, m}(\tau, \eta)}{s_{c, n, 1}(\tau, \eta)} \\
& \cdot \exp \left\{j \frac{4 \pi\left(R_{n, m}(\eta) f_{c, m}-R_{n, 1}(\eta) f_{c, 1}\right)}{c}\right\} .
\end{aligned}
$$




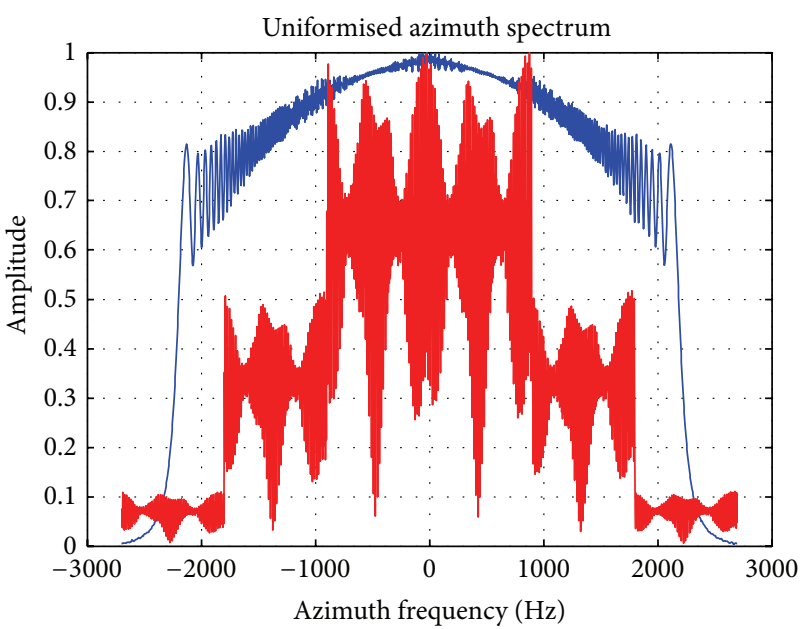

- No phase error

_ With phase error

(a)

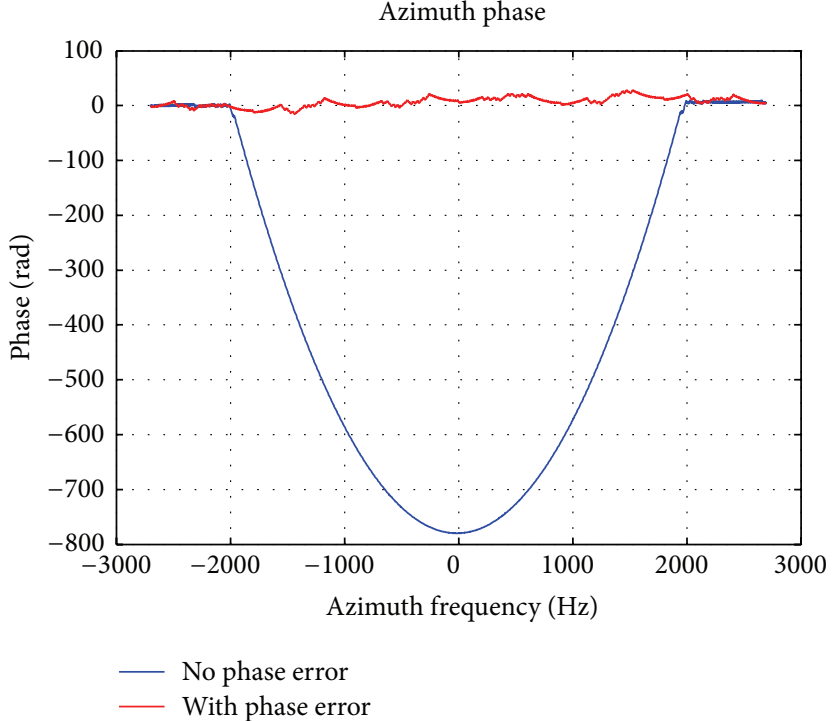

(b)

Figure 3: Results of azimuth reconstruction. (a) Azimuth spectrum. (b) Phase.

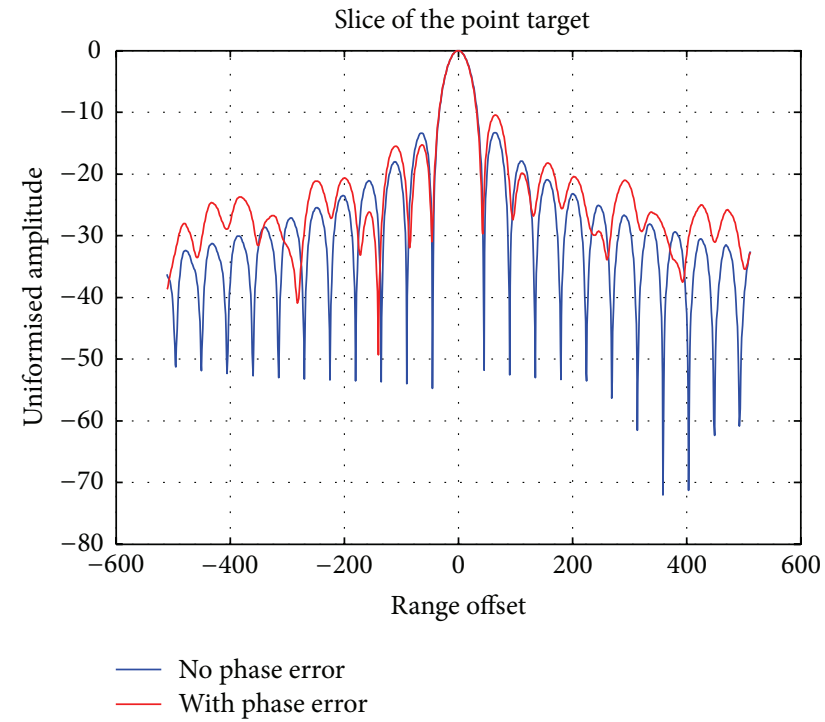

FIGURE 4: Results of subband synthesis.

To make the estimation more precisely and fast, not the whole image but parts of the image with high signal to cluster ratio (SCR) are used. A rectangular sliding window of appropriate size is traveled throughout the image, with the variance of the pixels in the window calculated, those high SCR targets are likely to exist in the windows with high variance [23].

\section{Simulation}

In this Section, point target simulations are introduced to analyze the proposed algorithm. The simulation parameters are listed in Table 1 . The system has 6 channels in azimuth

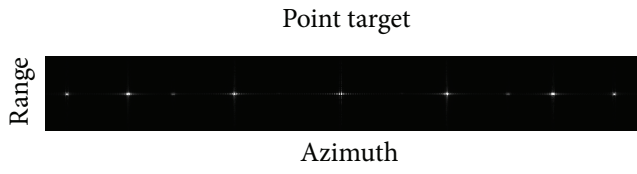

(a)

Point target

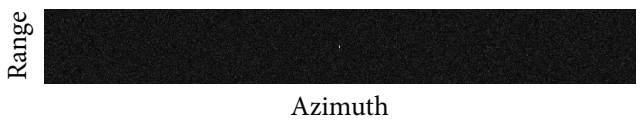

(b)

FIGURE 5: Imaging of the target point. (a) With phase errors. (b) Without phase errors.

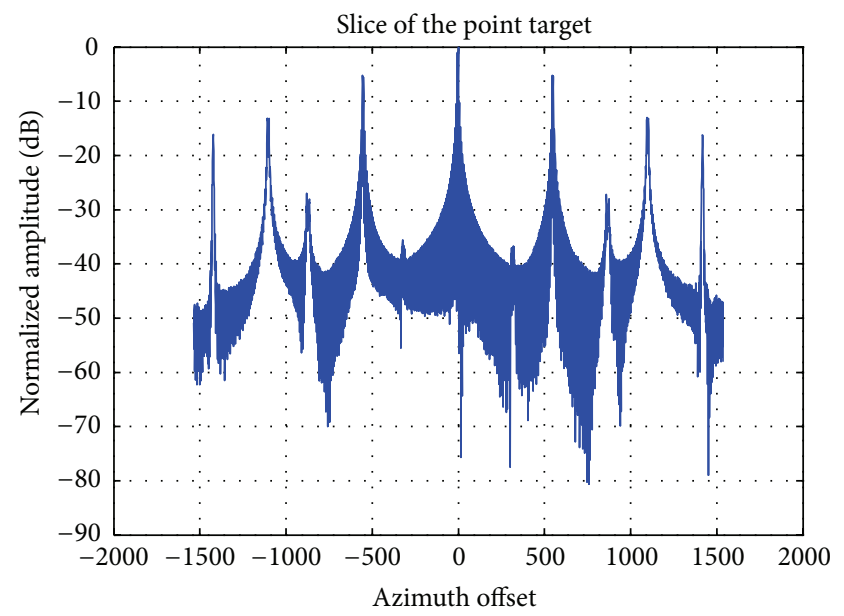

FIgure 6: Slice of the point target in Figure 5(a). 


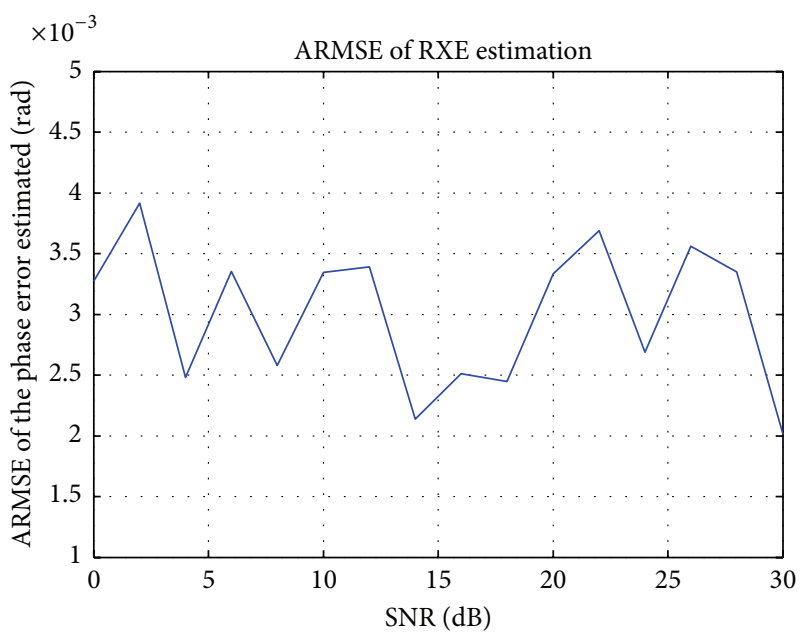

(a)

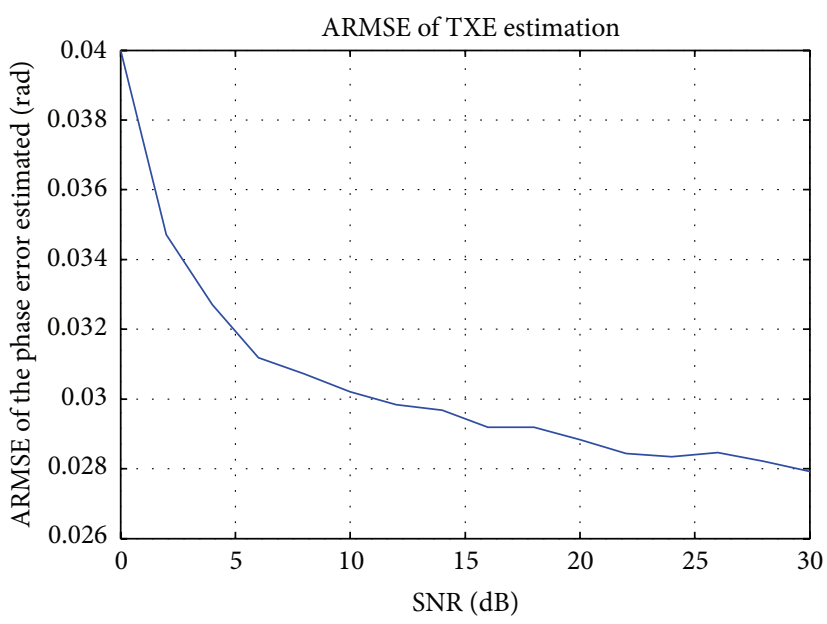

(b)

Figure 7: Results of Monte Carlo simulation. (a) Results of RXE estimation. (b) Results of TXE estimation.

TABLE 1: Simulation parameters.

\begin{tabular}{lc}
\hline Parameter & Value \\
\hline Channels & 6 \\
Center frequency & $9.35 \mathrm{GHz} \sim 9.85 \mathrm{GHz}$ \\
Chirp bandwidth & $100 \mathrm{MHz}$ \\
Pulse length & $20 \mathrm{us}$ \\
Platform velocity & $7000 \mathrm{~m} / \mathrm{s}$ \\
PRF & $900 \mathrm{~Hz}$ \\
Antenna length (full) & $6 \mathrm{~m}$ \\
Range & $800 \mathrm{~km}$ \\
\hline
\end{tabular}

with center frequency ranged from $9.35 \mathrm{GHz}$ to $9.85 \mathrm{GHz}$ stepped by $100 \mathrm{MHz}$, in which the overlapped ratio of subband in range frequency is zero. The full antenna length is $6 \mathrm{~m}$ in azimuth which means each aperture can share one out of six.

First, impacts of the phase error on azimuth reconstruction as well as range matched filtering are confirmed. Figure 3 gives the results of azimuth reconstruction. The RXEs added are $0.030 \pi,-0.055 \pi, 0.041 \pi,-0.035 \pi$, and $-0.018 \pi$, respectively. It is clear that the energy is confused across the spectrum as the existence of RXE. And the most important influence is that the phase no longer holds the form of quadratic which means the azimuth compression will fail. Figure 4 gives the results of subband synthesis. The TXEs added are $0.50 \pi,-0.55 \pi,-0.21 \pi, 0.09 \pi$, and $-0.47 \pi$, respectively. As mentioned in Section 3.2, the sidelobe indicators are worsened that the shape is distorted and no longer symmetrical, whereas the mainlobe remains the same; that is, the range resolution is not declined. Comparing the order of RXE with that of TXE, it can be found that azimuth reconstruction is more sensitive to the phase error.

Second, the validation of the proposed algorithm is verified. The system SNR is set to $20 \mathrm{~dB}$. As only point target is involved in the simulations, the computation cost of frequency domain imaging algorithm is acceptable. Thus the chirp scaling algorithm is used. Figure 5 gives the imaging results. The ghost targets are clearly seen on Figure 5(a) and Figure 6 in which the real target is defocused without doubt. After the compensation, the ambiguity is suppressed; see Figure 5(b). The estimation results are given in Table 2.

At last, the performance of the proposed algorithm is investigated using Monte Carlo experiment. The TXE interval as well as that of RXE is set to $[-0.5 \pi, 0.5 \pi]$ with uniform distribution. The system SNR is set from $0 \mathrm{~dB}$ to $30 \mathrm{~dB}$ with step of $2 \mathrm{~dB}$. For each SNR, the experiment is carried out 100 times. The experiment results are given by Figure 7, where the averaged root-mean-square error (ARMSE) is defined by

$$
\operatorname{ARMSE}=\frac{1}{M-1} \sum_{m=2}^{M}\left(\frac{1}{N} \sqrt{\sum_{n=1}^{N}\left(\widehat{\varepsilon}_{m, n}-\varepsilon_{m}\right)^{2}}\right),
$$

where $M$ is the total channels, $N$ is the simulation times, $\varepsilon$ is the error set and $\widehat{\varepsilon}$ is the error estimated, and substrip $m$ and $n$ indicate the channel and the sample, respectively. From Figure 6, it is shown that ARMSE of RXE estimation is almost independent on system SNR. This is because the cost function is obtained from SAR image where the energy is focused, and noise is greatly suppressed by the defined cost function ISCA (10). However, ARMSE of TXE estimation is dependent on system SNR which means that (16) has room for improvement. The ARMSE order of TXE is bigger than that of RXE because the estimation of RXE is prior to that of TXE; that is, the estimation error propagates.

\section{Conclusion}

MIMO technique offers an opportunity to map wider image swaths with improved spatial resolution in SAR system. Aiming at the inevitable channel imbalance of multichannel system, this paper has described a new algorithm for compensating the phase errors in MIMO-SAR systems based on 
TABLE 2: The phase errors added and estimated.

\begin{tabular}{lcccccccccc}
\hline & \multicolumn{3}{c}{ TXE } & \multicolumn{1}{c}{ RXE } \\
Channel & 2 & 3 & 4 & 5 & 6 & 2 & 3 & 4 & 5 & 6 \\
\hline Error & $0.50 \pi$ & $-0.55 \pi$ & $-0.21 \pi$ & $0.09 \pi$ & $-0.47 \pi$ & $0.030 \pi$ & $-0.055 \pi$ & $0.041 \pi$ & $-0.035 \pi$ & $-0.018 \pi$ \\
Estimation & $0.512 \pi$ & $-0.601 \pi$ & $-0.213 \pi$ & $0.078 \pi$ & $-0.455 \pi$ & $0.0321 \pi$ & $-0.0564 \pi$ & $0.0440 \pi$ & $-0.0372 \pi$ & $-0.0203 \pi$ \\
\hline
\end{tabular}

raw data. Compared with the internal calibration, not only the transmitter and the receiver phase imbalances but also the antenna phase imbalances can be removed. The phase error is classified as transmit phase error or receive phase error which is compensated separately. Simulations confirm the contribution of different kinds of error to image quality decline. Meanwhile the performance with different SNR is also investigated. Algorithm considering gain errors will be our future research focus.

\section{Conflict of Interests}

The authors declare that there is no conflict of interests regarding the publication of this paper.

\section{References}

[1] J. C. Curlander and R. N. McDonough, Synthetic Aperture Radar-Systems and Signal Processing, John Wiley \& Sons, Hoboken, NJ, USA, 1991.

[2] J. Klare, A. Brenner, and J. Ender, "A new airborne radar for 3D imaging-Image formation using the ARTINO principle," in Proceedings of the 6th European Conference on Synthetic Aperture Radar (EUSAR '06), pp. 16-18, 2006.

[3] A. Currie and M. A. Brown, "Wide-swath SAR," IEE Proceedings F: Radar and Signal Processing, vol. 139, no. 2, pp. 122-135, 1992.

[4] J.-H. Kim, M. Younis, P. Prats-Iraola, M. Gabele, and G. Krieger, "First spaceborne demonstration of digital beamforming for azimuth ambiguity suppression," IEEE Transactions on Geoscience and Remote Sensing, vol. 51, no. 1, pp. 579-590, 2013.

[5] W. G. Carrara, R. S. Goodman, and R. M. Majewski, Spotlight Synthetic Aperture Radar, Artech House, Norwood, Mass, USA, 1995.

[6] J. Mittermayer, A. Moreira, and O. Loffeld, "Spotlight SAR data processing using the frequency scaling algorithm," IEEE Transactions on Geoscience and Remote Sensing, vol. 37, no. 5, pp. 2198-2214, 1999.

[7] R. K. Moore, J. P. Claassen, and Y. H. Lin, "Scanning spaceborne synthetic aperture radar with integrated radiometer," IEEE Transactions on Aerospace and Electronic Systems, vol. 17, no. 3, pp. 410-421, 1981.

[8] P. Prats, R. Scheiber, J. Mittermayer, A. Meta, and A. Moreira, "Processing of sliding spotlight and TOPS SAR data using baseband azimuth scaling," IEEE Transactions on Geoscience and Remote Sensing, vol. 48, no. 2, pp. 770-780, 2010.

[9] W. Xu, P. Huang, R. Wang, and Y. Deng, "Processing of multichannel sliding spotlight and TOPS synthetic aperture radar data," IEEE Transactions on Geoscience and Remote Sensing, vol. 51, no. 8, pp. 4417-4429, 2013.

[10] W.-Q. Wang, "Space-time coding MIMO-OFDM SAR for highresolution imaging," IEEE Transactions on Geoscience and Remote Sensing, vol. 49, no. 8, pp. 3094-3104, 2011.
[11] W.-Q. Wang, "Virtual antenna array analysis for MIMO synthetic aperture radars," International Journal of Antennas and Propagation, vol. 2012, Article ID 587276, 10 pages, 2012.

[12] S. Yuepeng and Y. Ruliang, "High resolution, wide swath SAR using sub-aperture sub-band technique," in Proceedings of the CIE International Conference on Radar (ICR '06), Shanghai, China, October 2006.

[13] J. H. G. Ender and J. Klare, "System architectures and algorithms for radar imaging by MIMO-SAR," in Proceedings of the IEEE Radar Conference, Pasadena, Calif, USA, May 2009.

[14] G. Krieger, N. Gebert, and A. Moreira, "Unambiguous SAR signal reconstruction from nonuniform displaced phase center sampling," IEEE Geoscience and Remote Sensing Letters, vol. 1, no. 4, pp. 260-264, 2004.

[15] W. Jing, M. Xing, C.-W. Qiu, Z. Bao, and T.-S. Yeo, "Unambiguous reconstruction and high-resolution imaging for multiplechannel SAR and Airborne experiment results," IEEE Geoscience and Remote Sensing Letters, vol. 6, no. 1, pp. 102-106, 2009.

[16] R. T. Lord and M. R. Inggs, "High resolution SAR processing using stepped-frequencies," in Proceedings of the IEEE International Geoscience and Remote Sensing Symposium (IGARSS '97), pp. 490-492, August 1997.

[17] F. Berizzi and G. Corsim, "Autofocusing of inverse synthetic aperture radar images using contrast optimization," IEEE Transactions on Aerospace and Electronic Systems, vol. 32, no. 3, pp. 1185-1191, 1996.

[18] C. G. Gao, Study on new techniques for high-resolution and wide-swath synthetic aperture radar [Ph.D. thesis], University of Chinese Academy of Sciences, 2012.

[19] J. K. Thomas, "Monotonic iterative algorithm for minimumentropy autofocus," in Proceedings of the Adaptive Sensor Array Processing Workshop (ASAP '06), June 2006.

[20] I. G. Cumming and F. H. Wong, Digital Signal Processing of Synthetic Aperture Radar Data: Algorithms and Implementation, Artech House, Norwood, Mass, USA, 2004.

[21] M. D. Desai and W. K. Jenkins, "Convolution backprojection image reconstruction for spotlight mode synthetic aperture radar," IEEE Transactions on Image Processing, vol. 1, no. 4, pp. 505-517, 1992.

[22] Q. Chen, Study on new techniques for high-resolution and wide-swath spaceborne synthetic aperture radar [Ph.D. thesis], University of Chinese Academy of Sciences, 2013.

[23] R. C. Gonzalez and R. E. Woods, Digital Image Processing, Prentice Hall, 3rd edition, 2007. 

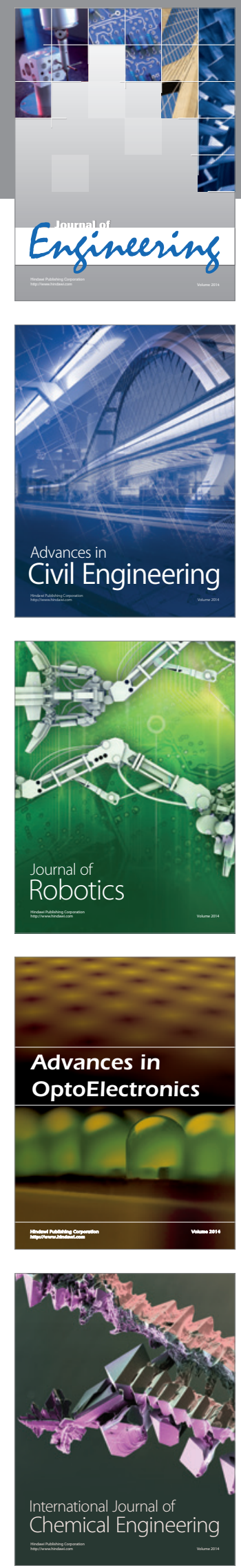

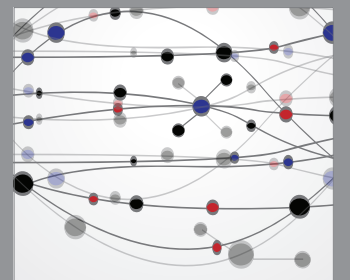

The Scientific World Journal
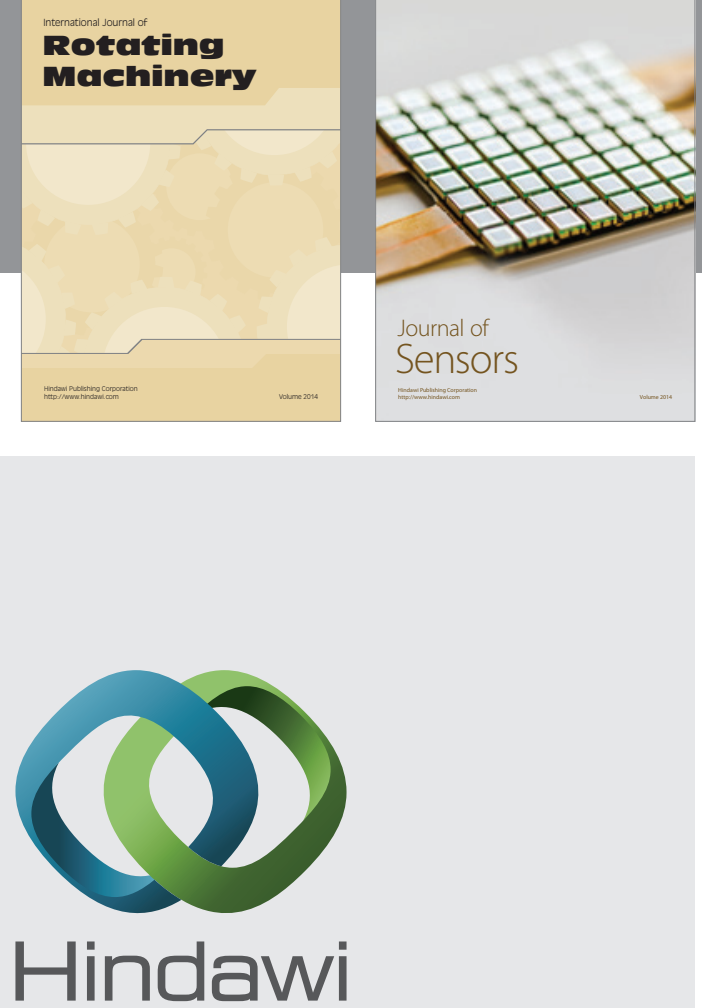

Submit your manuscripts at http://www.hindawi.com
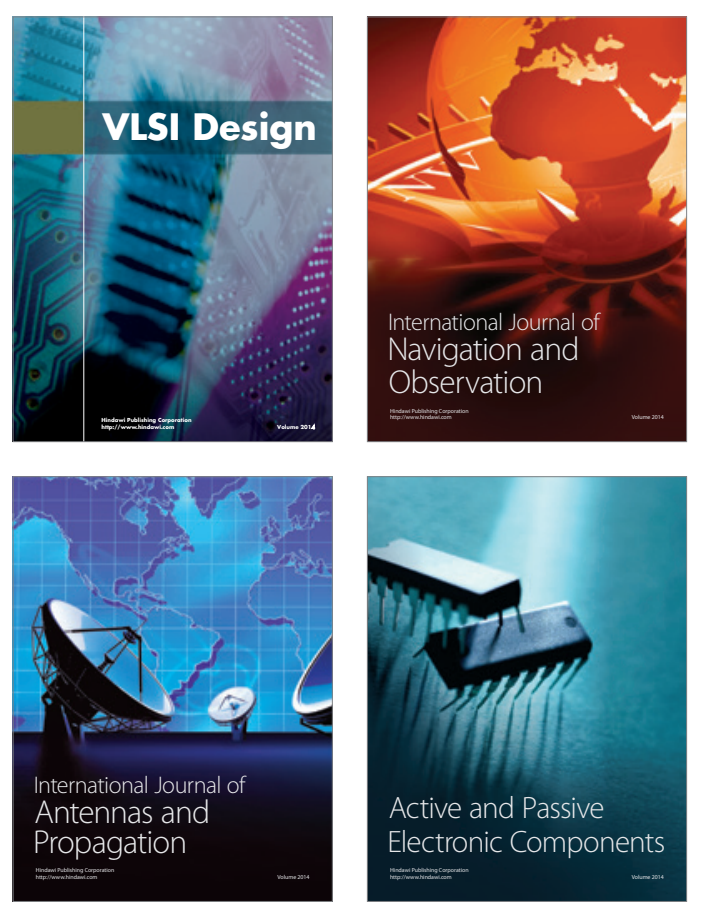
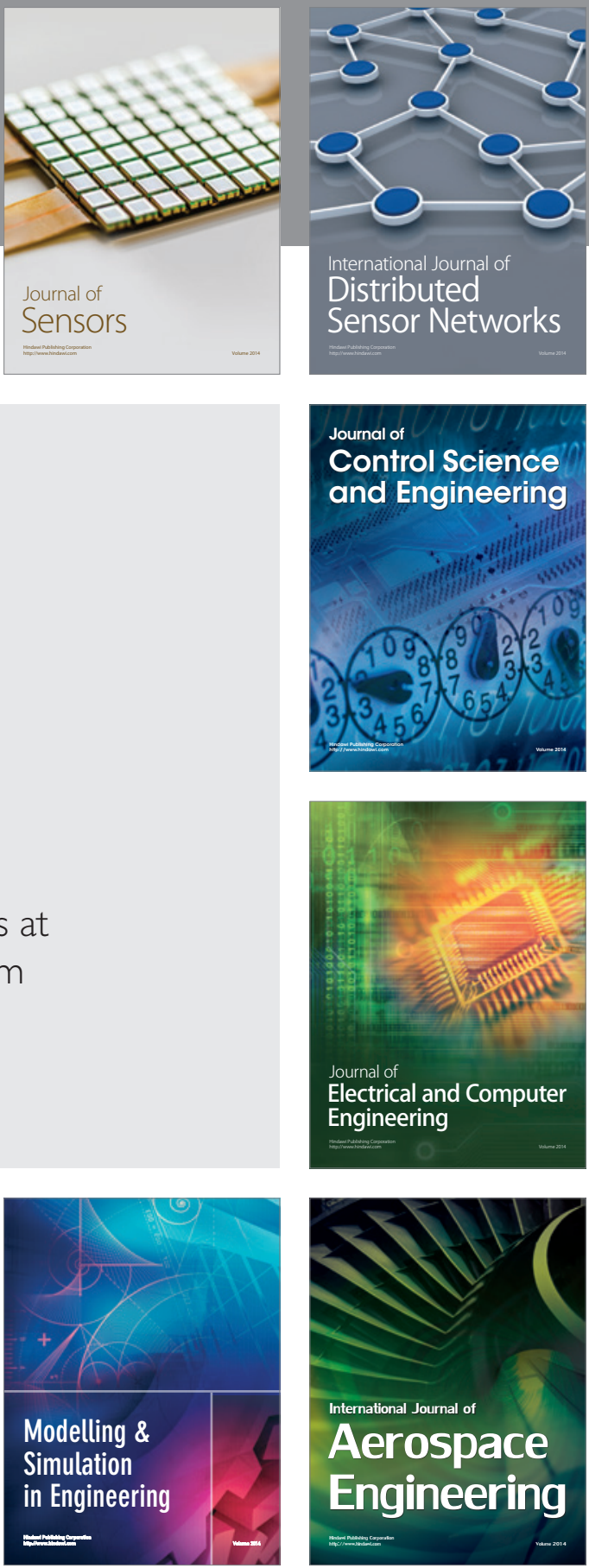

Journal of

Control Science

and Engineering
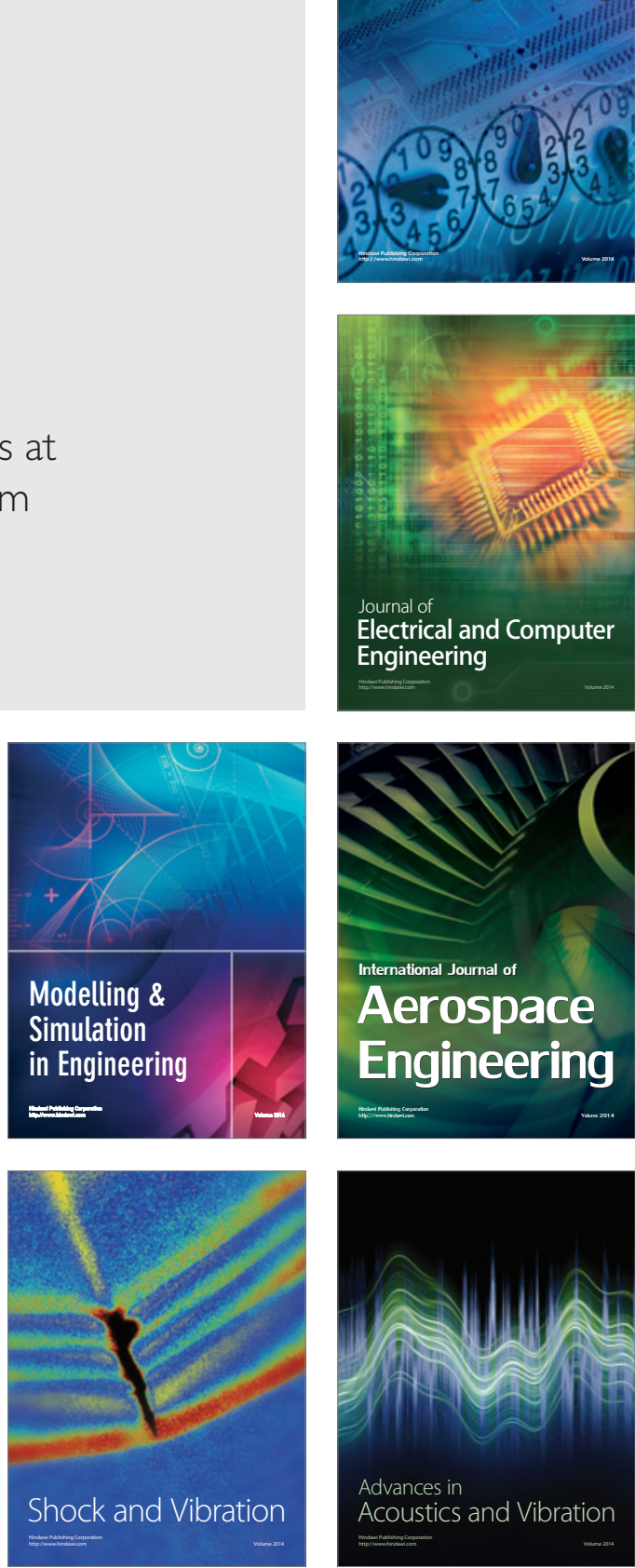